\title{
High Expressions of CUL4A and TP53 in Colorectal Cancer Predict Poor Survival
}

\author{
Chao Lia Juyuan Bu ${ }^{b}$ Yifeng Liao ${ }^{c}$ Jin Zhang ${ }^{a}$ Jun Han ${ }^{a}$ Han Zhang ${ }^{a}$ \\ Hao Xing ${ }^{a}$ Zhenli Li ${ }^{a} \quad H_{a n}$ Wu $^{a}$ Lei Liang ${ }^{a}$ Mingda Wang ${ }^{a}$ Wenxing Qin ${ }^{d}$ \\ Tian Yang ${ }^{\mathrm{a}}$
}

aDepartment of Hepatobiliary Surgery, Eastern Hepatobiliary Surgery Hospital, Second Military Medical University, Shanghai, ' ${ }^{b}$ epartment of gastrointestinal surgery, the Fifth Affiliated Hospital of Sun YatSen University, Guangzhou, 'Department of abdominal chemotherapy, the Fifth Affiliated Hospital of Sun Yat-Sen University, Guangzhou, dDepartment of Clinical Oncology, Changzheng Hospital, Second Military Medical University, Shanghai, China

\section{Key Words \\ CUL4A • Colorectal cancer $•$ EMT $・$ Prognosis $\cdot$ ZEB1}

\begin{abstract}
Background/Aims: Cullin 4A (CUL4A) is vital in cell survival, development, growth and cell cycle, it plays an important role in chaperone-mediated ubiquitination and interacts with TP53 in carcinogenesis. However, the clinicopathologic significance of CUL4A expression in colorectal cancer is unknown; in particular, the prognostic value of CUL4A combined with TP53 expression has not been explored. Methods: We analyzed the expression of CUL4A in both public database (Oncomine) and 180 cases of colorectal cancer and paired normal tissues by real-time polymerase chain reaction and western blotting. Colony formation, wound healing, migration and invasion assays and tumorigenesis in nude mice were used to explore the function of CUL4A in CRC proliferation and metastasis in vitro and in vivo. Markers of epithelial to mesenchymal transition (EMT) were evaluated by western blotting. Immunohistochemistry (IHC) was used to analyse the relationship between CUL4A expression and E-cadherin expression. Results: CUL4A and TP53 protein expression was significantly higher in cancerous tissues compared to normal tissues. Significant correlation between CUL4A and TP53 expression was observed. CUL4A expression was an independent prognostic factor for overall survival (OS) and disease-free survival (DFS). Interestingly, patients with tumors that had both CUL4A overexpression and mutant TP53 protein accumulation relapsed and died within a significantly short period after surgery $(P<0.001)$. Multivariate analysis showed that patients with both CUL4A+ and TP53 + positive tumors had extremely poor OS and DFS. Knockdown of CUL4A by a short interfering RNA (siRNA) significantly suppressed the progression of EMT, proliferation, migration, and invasion of colon cancer cells in vitro and tumor growth in vivo. ZEB1 silencing blocked CUL4A-driven these processes. Conclusion: CUL4A expression correlated positively

C. Li, J. Bu, Y. Liao, J. Zhang and J. Han contributed equally to this work.

\begin{tabular}{ll}
\hline Tian Yang, MD & Dept. of Hepatobiliary Surgery, Eastern Hepatobiliary Surgery Hospital, Second Military Medical University; \\
and Wenxing Qin, MD & $\begin{array}{l}\text { Dept. of Clinical Oncology, Changzheng Hospital, Second Military Medical University } \\
\text { Shanghai } 20438 \text { (China), E-Mail yangtiandfgd@hotmail.com; oliverqwx@139.com }\end{array}$
\end{tabular}
\end{abstract}




\section{Cellular Physiology Cell Physiol Biochem 2018;51:2829-2842

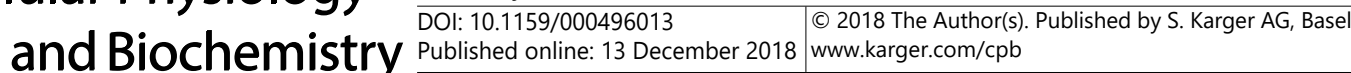 \\ Li et al.: CUL4A and TP53 in Colorectal Cancer}

with the prognosis of colorectal cancer. Mechanistically, ZEB1 was confirmed to mediate the function of CUL4A in regulating the EMT. The assessment of both CUL4A and mutant TP53 expression will be helpful in predicting colon cancer prognosis.

\section{Introduction}

Colorectal adenocarcinoma is affecting approximately 112, 000 new patients every year [1]. Despite improvements in surgical and adjuvant chemotherapy treatment, 5-year survival rates from CRC still remain unsatisfactory [2,3]. Sensitive biomarkers are crucial for early diagnosis and predicting prognosis $[4,5]$. Although several independent clinical prognostic factors for CRC have been identified, increasing evidence suggests that it is insufficient for predicting the clinical outcome of these patients [6-8]. To improve clinical outcome of colorectal cancer patients, novel molecular prognostic markers are needed as well as improved understanding of the mechanism of tumorigenesis.

Cul4A belongs to the family of evolutionally conserved cullin proteins, including seven related cullins (Cul1, Cul2, Cul3, Cul4A, Cul4B, Cul5 and Cul7) [9]. Cul4A is an important component of $\mathrm{E} 3$ ubiquitin ligase family and ubiquitinates a large number of substrates such as DDB2, REDD1 and histone H2A [10-12]. Changes in CUL4A potentially exert pleiotropic effects that alter cellular functions, including proliferation, differentiation, and apoptosis [13-15]. CUL4A mRNA and protein levels are highly correlated with 13q34 amplification and have been hypothesized to be the candidate biomarker in breast cancers and hepatocellular carcinomas $[16,17]$, as well as in other cancers, such as lung cancer, prostate cancer and ovarian carcinoma [18-20], further supporting its possible role in the aggressive behavior of certain cancers. However, there have been no reports concerning CUL4A protein expression in CRC and its association with clinical outcome.

TP53 is one of the most important tumor suppressor genes, mutated in over 50\% of human malignancies [21-23]. It regulates DNA repair, cell cycle and apoptosis and therefore plays an essential role in maintaining genetic stability [22]. Mutations of TP53, which are considered to be able to bear ubiquitination, are thought to be involved in the pathogenesis of as many as $60 \%$ of colon cancers [24-26]. With a prolonged half-life, TP53 protein detected by immunohistochemistry assay has been widely used as a detection method for TP53 mutation [27-29]. Although it has been suggested that TP53 plays a critical role in cancer progression $[30,31]$, to our knowledge no published data on the correlation between mutants TP53 and CUL4A, especially the prognostic value of their combined expression pattern exist so far.

The local or distant invasion and metastasis is one of the most common causes that leads to the poor prognosis in colorectal cancer patients, however, the molecular mechanism is still not fully elucidated. Epithelial-mesenchymal transition (EMT) resulted in the loss of cell polarity and tight junction between cells, which allowed the epithelial cells to get the invasion and migration ability. EMT is an effective way to acquire the migration ability in epithelial cells, so it has become an important approach for the invasion and metastasis of epithelial cell carcinoma. EMT cells strongly promoted the expression of MMP family protein including MMP1, MMP2, and MMP7 through the upregulation of Snail and SIPI in primary hepatic carcinoma [32]. ZEB1 performs a significant role as an regulatory molecule in the process of carcinoma cells epithelial-mesenchymal transition (EMT), which bring about tumor invasion and metastasis. Studying the regulation of proliferation and EMT will greatly benefit our understanding of colorectal cancer.

In the present study, we analyzed CUL4A and TP53 expression by immunohistochemistry analysis in both normal and malignant colorectal tissues using tissue microarrays. We correlated CUL4A and TP53 expression with clinicopathological characteristics as well as survival rate in patients with colorectal cancers. Our findings indicated CUL4A overexpression 


\section{Cellular Physiology Cell Physiol Biochem 2018;51:2829-2842

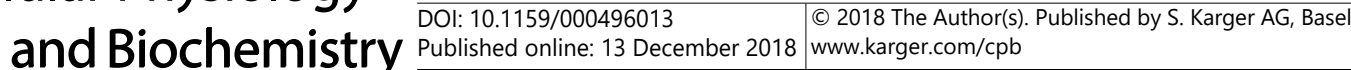

Li et al.: CUL4A and TP53 in Colorectal Cancer

promoted proliferation and EMT in colorectal cancer cells via ZEB1, resulting in enhancement of growth, migration, and invasion in vitro and metastasis in vivo.

\section{Materials and Methods}

\section{Human tissue specimens and patient information}

A total of 180 paired cancer and matched normal surgical margins of formalin-fixed paraffinembedded (FFPE) tissue samples were collected. All tissue blocks were obtained from the department of general surgery, Shanghai General Hospital from 2006 to 2010. Clinical characteristics of cancer patients were extracted from their medical record, including: age, sex, histological type, differentiation grade and tumor stage. None of the cancer patients received any types of treatments (radiation therapy, chemotherapy, or immunotherapy) before surgery. The patients' disease-free survival (DFS) and overall survival (OS) durations were defined as the interval from initial surgery to clinically or radiologically proven recurrence or metastasis and from initial surgery to death, respectively. The study protocol was approved by the Human Research Ethics Committee and all patients signed an informed consent for the provision of biological material for future research purposes.

Tissue microarray (TMA) construction and immunohistochemistry analysis (IHC)

After screening hematoxylin and eosin (H\&E)-stained slides for optimal tumor tissue and tumor adjacent tissue up to $2 \mathrm{~cm}$ from the tumor, TMA slides were constructed (in collaboration with Shanghai Biochip). Specifically, core tissue biopsies (2 $\mathrm{mm}$ in diameter) were taken from individual FFPE blocks and arranged in a new recipient paraffin block. Four-micron sections were cut and placed on super frostcharged glass microscope slides to generate TMA slides. Tissue sections were deparaffinized and rehydrated through graded alcohols. Endogenous peroxidase activity was blocked by incubation in $3 \% \mathrm{H}_{2} \mathrm{O}_{2}$. Antigen retrieval was carried out with $0.01 \mathrm{M}$ citrate buffer $\mathrm{pH} 6.0$ and microwave heat induction. CUL4A was detected by rabbit polyclonal anti-human CUL4A antibody (dilution 1:500) (Abcam, ab72548), and TP53 was detected by rabbit monoclonal anti-human TP53 antibody (dilution 1:300) (Abcam, ab32049). The steps were performed using the Envision two step method. The Envision and DAB Color Kit was purchased from Gene Tech Company Limited (Shanghai). Counterstained with Hematoxylin, dehydrated through graded alcohols, cleared in xylene, and coverslipped with permanent mounting media. All cases were reviewed and scored without knowledge of clinical characteristics. The scoring system includes the extent and intensity of staining. Briefly, the intensity was assigned a score of $0,1,2$, or 3, representing negative, weak, moderate, or strong expression, respectively. Whereas, the extent was assigned a score of $0,1,2$, or 3 , representing negative, $<10 \%, 10 \%-50 \%$, and $>50 \%$ of cells stained. The overall quantitation of the IHC score was obtained by multiplying the average intensity and score of five different high-power fields $(\times 400$ magnification).

\section{Cell culture and transient transfection}

Two human colorectal cancer cell lines, HCT8 and HT29, were purchased from the Type Culture Collection of the Chinese Academy of Science (Shanghai, China). All cell lines were cultured in Dulbecco's Modified Eagle's Medium (DMEM, Gibco) supplemented with 10\% fetal bovine strum (FBS, Gibco), 1\% penicillin-streptomycin (Gibco) and grown in a humidified $37^{\circ} \mathrm{C}$ incubator with $5 \% \mathrm{CO}_{2}$.

Three short hairpin RNAs were designed and the most effective target sequence of the CUL4A gene short interfering RNA (siRNA) were 5'-CCAUCUGGGAUAUGGGAUUTT-3'. A scrambled siRNA (5'-CUCCGAACGUGUCACGUTT-3') was used as a negative control. HCT8 and HT29 cells were transfected using Lipofectamine 2000 (Invitrogen) according to the manufacturer's protocol. SiNC and SiZEB1 were purchased from GENEWIZ (Suzhou, China). Transfection was performed with Lipofectamine 2000 (Invitrogen, United States) according to the manufacturer's instructions. 


\section{Cellular Physiology Cell Physiol Biochem 2018;51:2829-2842 \begin{tabular}{l|l|l} 
and Biochemistry Published online: 13 December 2018 & $\begin{array}{l}\text { (c) } 2018 \text { The Author(s). Published by S. Karger AG, Basel } \\
\text { www.karger.com/cpb }\end{array}$ \\
\hline
\end{tabular} \\ Li et al.: CUL4A and TP53 in Colorectal Cancer}

\section{Quantitative real-time polymerase chain reaction ( $q P C R$ )}

Total RNA was isolated from 38 tumor tissues and corresponding normal mucosae following the manufacturer's instructions (Qiagen, Hilden, Germany). First-stand cDNA was synthesized from $1 \mu \mathrm{g}$ of total RNA according to PrimeScript/tm RT Master Mix (Takara, shiga, Japan). qPCR was performed on a ViiA ${ }^{\mathrm{TM}} 7$ Real-Time PCR System (Life Technology, USA) with SYBR ${ }^{\circledR}$ Premix DimerEraser $^{\mathrm{TM}}$ (Perfect Real Time) (Takara, Japan) in accordance with the manufacturer's instructions. The following primer sequences were used for qPCR: CUL4A forward 5'-CAGCGGCTCTGATTACAGACCTCG-3' and reverse 5'-GTCTTCACAGGCCTGACGCAGT-3', GAPDH forward 5'-GCTGGCGCTGAGTACGTCGT-3' and reverse 5'-ACGTTGGCAGTGGGGACACG-3'. The cycling conditions were as follows: initial denaturation 2 min at $95^{\circ} \mathrm{C}$ followed 40 cycles of denaturation $\left(30 \mathrm{~s}\right.$ at $\left.95^{\circ} \mathrm{C}\right)$, annealing $\left(45 \mathrm{~s}\right.$ at $\left.61^{\circ} \mathrm{C}\right)$, and elongation $\left(45 \mathrm{~s}\right.$ at $\left.72{ }^{\circ} \mathrm{C}\right)$.

\section{Western blot analysis}

Protein was extracted from tissue samples and cells using RIPA lysis buffer. The protein concentration was measured using the BCA protein assay kit (Beyotime Biotechnology, China). The standard western blot was performed in order to transfer protein onto PVDF membranes. Membranes were then incubated with an antibody against CUL4A (1:200, Abcam, UK), E-cadherin (1:100, Abcam, UK), N-cadherin (1:100, Abcam, UK), Vimentin (1:100, Abcam, UK), ZEB1 (1:500, Abcam, UK) and GAPDH (1:1000, Cell Signaling Technology, USA) overnight at $4^{\circ} \mathrm{C}$. After washing with TBST, the membranes were incubated with a secondary antibody against mouse immunoglobulin G. The proteins were detected using ECL (Pierce Biotechnology, USA) according to the manufacturer's instructions.

\section{Colony formation assay}

Preparing single-cell suspension solutions and then plating on a 6-well plate with 500 cells per well evaluated colony-formation ability. After 14 days, the developed colonies were stained with Giemsa for 20 min, which were then counted and photographed.

\section{Cell scratch-wound assay}

For cell migration, colorectal cancer cells were seeded in six-well plates. A cell scratch-wound was generated using a $10-\mu \mathrm{L}$ tip. The wounded cells were photographed for measuring gap sizes at $0 \mathrm{~h}$ and $24 \mathrm{~h}$.

\section{Migration and invasion assays}

The invasion and migration ability of treated colorectal cancer cells were evaluated by using invasion assay and Transwell assay. Cells $\left(5 \times 10^{4}\right)$ were seeded into the upper chambers with Matrigel $(\mathrm{BD}, \mathrm{CA}$, USA), and the lower chamber was filled with $10 \%$ FBS. After $24 \mathrm{~h}$ incubation, migrated cells on the lower membrane were fixed, stained and counted under a microscope.

\section{Tumorigenesis in nude mice}

All protocols for the animal studies were approved by Shanghai Jiao Tong University affiliated Shanghai First People's Hospital Animal Care guidelines. SiCUL4A cells and controls $\left(1 \times 10^{6} ; \mathrm{n}=5 /\right.$ group $)$ were subcutaneously injected into nude mice (6-week-old) respectively. Four weeks later, tumor volume and weight were measured.

\section{Statistical analysis}

For statistical analysis, the continuous CUL4A and TP53 expression data from IHC were first converted into dichotic data (low vs high) using specific cutoff values, which were selected to be significant in terms of survival using the X-tile software program (The Rimm Lab at Yale University; http://www.tissuearray.org/ rimmlab). Student t test and Pearson $\chi 2$ test were used to determine the statistical significance of differences between comparison groups. The correlation between CUL4A and TP53 protein expression was calculated using Spearmans test. The cumulative patient survival was estimated using the Kaplan-Meier method, and a log-rank test was used to compare the survival curves. A Cox proportional hazards model was used to calculate multivariate hazard ratios for the variables. A P-value of less than 0.05 was considered statistically significant. All statistical analyses were carried out using the SPSS 19.0 statistical software package (SPSS Inc., Chicago, IL). 


\section{Cellular Physiology Cell Physiol Biochem 2018;51:2829-2842 and Biochemistry \begin{tabular}{l|l} 
DOI: 10.1159/000496013 & $\begin{array}{l}\text { @ } 2018 \text { The Author(s). Published by S. Karger AG, Basel } \\
\text { wublished }\end{array}$
\end{tabular} \\ Li et al.: CUL4A and TP53 in Colorectal Cancer}

\section{Results}

\section{CUL4A or TP53 expression in colorectal tissues}

Using the X-tile software program for TMA data analysis (http://www.tissuearray. org/rimmlab), we first identified significant cutoff point in terms of overall survival in colorectal cancers. For CUL4A, the cutoff 7 was selected for colorectal cancers: score 0-7 was considered low expression while 8-9 was considered high expression. For TP53, the cutoff point 7 was selected for colorectal cancer. For all subsequent analyses, CUL4A and TP53 protein expression levels were considered either as Low or High using these cutoff values. Of the 180 specimens on the paired TMA, 155 (86.1\%) showed CUL4A low staining in normal mucosa. In contrast, CUL4A was prominently expressed in colon cancerous tissue specimens, with 95 (52.8\%) of cancerous tissues had high CUL4A expression (CUL4A+), significantly higher than normal surgical margin tissues (Table 1). Similarly, the frequency of high TP53 expression (TP53+) was significantly higher in cancers than normal surgical margins and benign lesions. Interestingly, high CUL4A and high TP53 expression (CUL4A+/TP53+) was almost exclusively present in cancerous tissues, only 3 cases in colorectal normal tissues had CUL4A+/TP53+ staining. We also found that tumor differentiation was significantly correlated with the expression of CUL4A and TP53 (Fig. 1C).

In order to confirm the expression of CUL4A in colorectal cancer, we first primarily browse Oncomine (www.oncomine.org) to acquire CUL4A expression in colorectal cancer, then we discovered that its level was obviously higher in tumor tissues compared with normal tissues (Fig. 1A). we performed western blotting and qPCR analysis. These experimental results showed that CUL4A expression level was higher in cancerous tissues than that in the adjacent normal tissues both at the transcriptional and post-transcriptional levels (Fig. 1B). These data suggested that the expression of CUL4A and TP53 is closely related to the occurrence and development of colorectal cancer.

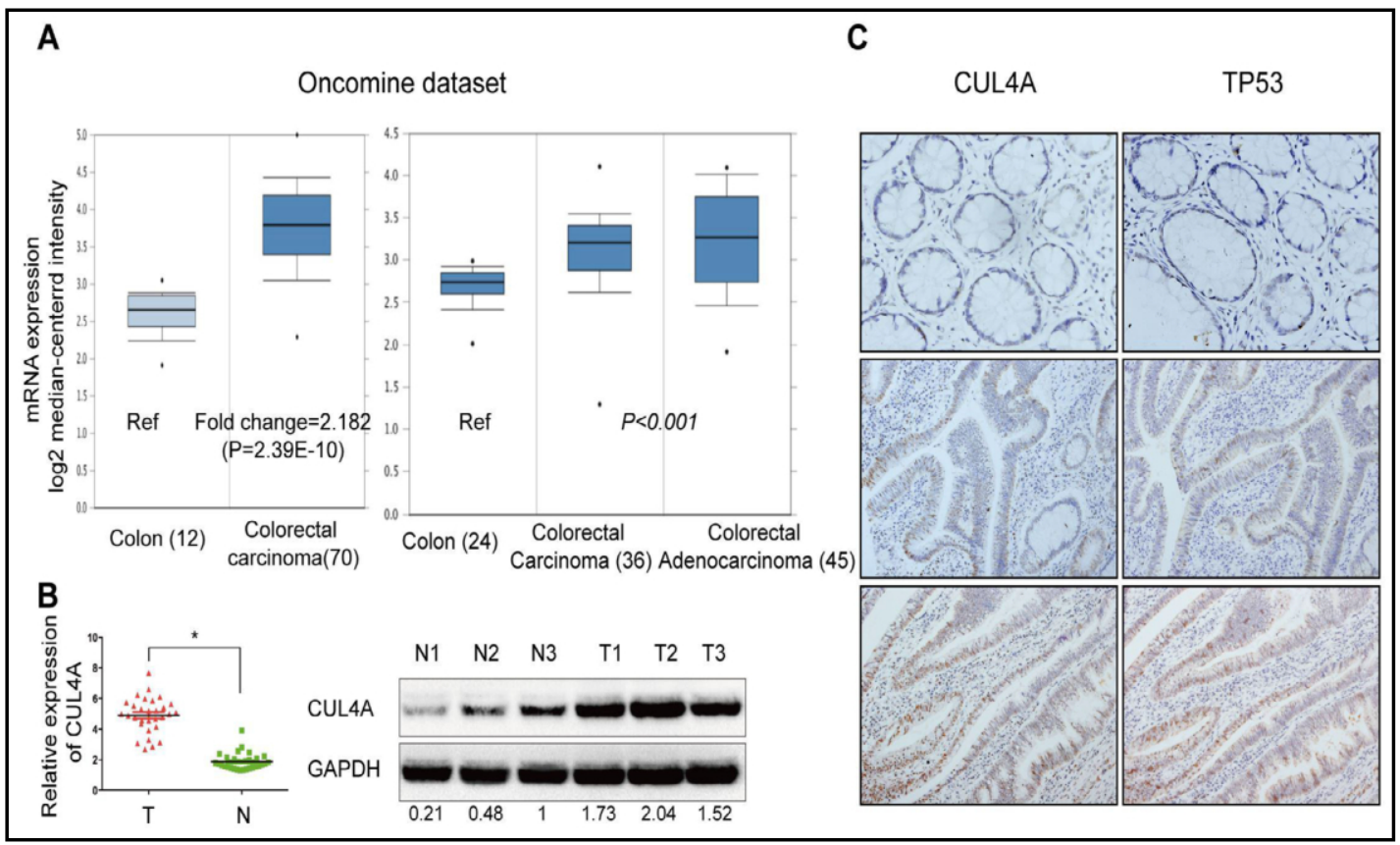

Fig. 1. Representation of CUL4A expression in colorectal normal and malignant tissues. (A) Exhibition of CUL4A expression of colorectal carcinoma and normal specimens from colorectal statistics in Oncomine dataset. (B) Expression of CUL4A in colorectal cancer and adjacent normal tissues. (C) Representation of CUL4A and TP53 protein expression in colorectal normal and malignant tissues on TMA sections. Original magnification $\times 200$. 
Table 1. CUL4A, TP53 and ZEB1 expression in colorectal cancer tissues. ${ }^{*} \mathrm{P}<0.05$ indicates a significant association among the variables. ${ }^{* *}$ The significant difference of correlation between CUL4A and TP53 based on Spearman test

\begin{tabular}{|c|c|c|c|c|c|c|c|c|c|c|}
\hline \multirow[b]{2}{*}{ Parameter } & \multirow[b]{2}{*}{ Cases } & \multicolumn{2}{|c|}{ CUL4A expression } & \multirow[t]{2}{*}{$P$ value } & \multicolumn{2}{|c|}{ TP53 expression } & \multirow[t]{2}{*}{$\mathrm{P}$ value } & \multicolumn{2}{|c|}{ ZEB1 expression } & \multirow[t]{2}{*}{$P$ value } \\
\hline & & Low(85) & High(95) & & Low(92) & High(88) & & low(83) & high(97) & \\
\hline Age (years) & & & & 0.593 & & & 0.973 & & & 0.124 \\
\hline$<65$ & 80 & 36 & 44 & & 41 & 39 & & 42 & 38 & \\
\hline$\geq 65$ & 100 & 49 & 51 & & 51 & 49 & & 41 & 59 & \\
\hline Sex & & & & 0.157 & & & 0.567 & & & 0.149 \\
\hline Male & 82 & 34 & 48 & & 40 & 42 & & 33 & 49 & \\
\hline Female & 98 & 51 & 47 & & 52 & 46 & & 50 & 48 & \\
\hline Location & & & & 0.455 & & & 0.766 & & & 0.881 \\
\hline Right & 90 & 40 & 50 & & 45 & 45 & & 42 & 48 & \\
\hline Others & 90 & 45 & 45 & & 47 & 43 & & 41 & 49 & \\
\hline $\begin{array}{l}\text { Tumor size } \\
(\mathrm{cm})\end{array}$ & & & & $0.048^{*}$ & & & 0.280 & & & $0.046^{*}$ \\
\hline$<5$ & 94 & 51 & 43 & & 48 & 46 & & 50 & 44 & \\
\hline$\geq 5$ & 86 & 34 & 52 & & 37 & 49 & & 33 & 53 & \\
\hline AJCC stage & & & & $0.026^{*}$ & & & 0.535 & & & $0.011^{*}$ \\
\hline I & 16 & 11 & 5 & & 12 & 4 & & 13 & 3 & \\
\hline II & 44 & 24 & 20 & & 28 & 16 & & 21 & 23 & \\
\hline III & 104 & 47 & 57 & & 64 & 40 & & 45 & 59 & \\
\hline IV & 16 & 3 & 13 & & 8 & 8 & & 4 & 12 & \\
\hline T stage & & & & 0.127 & & & 0.289 & & & 0.063 \\
\hline $\mathrm{T} 1$ & 16 & 12 & 4 & & 8 & 3 & & 5 & 11 & \\
\hline $\mathrm{T} 2$ & 27 & 11 & 16 & & 12 & 7 & & 14 & 13 & \\
\hline $\mathrm{T} 3$ & 66 & 29 & 37 & & 32 & 34 & & 24 & 42 & \\
\hline $\mathrm{T} 4$ & 71 & 33 & 38 & & 40 & 44 & & 40 & 31 & \\
\hline $\mathrm{N}$ stage & & & & $<0.001^{*}$ & & & 0.103 & & & $<0.001^{*}$ \\
\hline No & 99 & 60 & 39 & & 54 & 45 & & 58 & 41 & \\
\hline N1 & 51 & 20 & 31 & & 28 & 23 & & 20 & 31 & \\
\hline N2 & 30 & 5 & 25 & & 10 & 20 & & 5 & 25 & \\
\hline M stage & & & & & & & & & & \\
\hline Mo & 164 & 79 & 85 & 0.414 & 82 & 82 & 0.340 & 76 & 88 & 0.843 \\
\hline M1 & 16 & 6 & 10 & & 10 & 6 & & 7 & 9 & \\
\hline Differentiation & & & & $0.049 *$ & & & $0.002^{*}$ & & & $0.007 *$ \\
\hline Well & 93 & 52 & 41 & & 58 & 35 & & 53 & 40 & \\
\hline Moderate & 63 & 23 & 40 & & 28 & 35 & & 20 & 43 & \\
\hline Poor & 24 & 10 & 14 & & 6 & 18 & & 10 & 14 & \\
\hline TP53 & & & & $0.024 * *$ & & & & & & $0.023^{*}$ \\
\hline Low & 92 & 51 & 41 & & & & & 50 & 42 & \\
\hline High & 88 & 34 & 54 & & & & & 33 & 55 & \\
\hline
\end{tabular}

Association of CUL4A and TP53 expression with clinicopathologic characteristics in colorectal cancers

Next, we examined the correlation between CUL4A or TP53 protein expression and clinical parameters among colorectal cancer patients. In colorectal cancer, high CUL4A expression was significantly associated with tumor size $(\mathrm{P}=0.048)$, differentiation $(\mathrm{P}=$ $0.049)$, N stage $(P<0.001)$ and AJCC stage $(P=0.026)$; while high TP53 expression was associated with tumor differentiation $(P=0.002)$ (Table 1). Significant correlation between CUL4A and TP53 expression was detected $(\mathrm{P}=0.024)$.

Prognostic value of CUL4A and TP53 protein expression in colorectal cancer

In colorectal cancer, high CUL4A expression was significantly associated with poor survival. The disease-free survival (DFS) time was analyzed in 164 patients in stages I-III, who accepted radical colectomy. The proportion of patients who developed recurrence or metastasis from primary colon cancer after radical colectomy differed substantially between the CUL4A-high and CUL4A-low group (Fig. 2A). The risk of developing recurrence or distant metastases after radical colectomy was much higher in patients with CUL4A-high tumors (31 [37.8\%] of 82 patients, mean follow-up 65.8 months [range 62.5-73.5]) compared with patients with CUL4A-low tumors (12 [14.6\%] of 82, mean follow-up 75.0 months [range 71.8-82.5]) ( $\mathrm{P}<0.001)$. CUL4A could serve as a novel prognostic marker to predict risk of DFS in patients with radical colectomy (hazard ratio [HR] 1.87; 95\% confidence interval [CI] 1.19-3.53; P = 0.042, Table 2). Furthermore, Kaplan-Meier analysis also showed that the expression of CUL4A was significantly correlated with overall survival (OS) of colon cancer patients (log-rank test, $\mathrm{P}<0.001$, Fig. $2 \mathrm{~B}$ ). In multivariate analysis with clinicopathological variables, including age, gender, clinical stage and differentiation grade, the expression of CUL4A had an independent prognostic value to predict patients' outcomes (HR 4.06; 95\% CI 1.08-14.9; $\mathrm{P}=0.038$, Table 2). On the other hand, mutant TP53 had no relation with DFS but was significantly associated with $\mathrm{OS}(\mathrm{P}=0.031$; Fig. $2 \mathrm{C}$ and $2 \mathrm{D})$. Notably, although mutant 


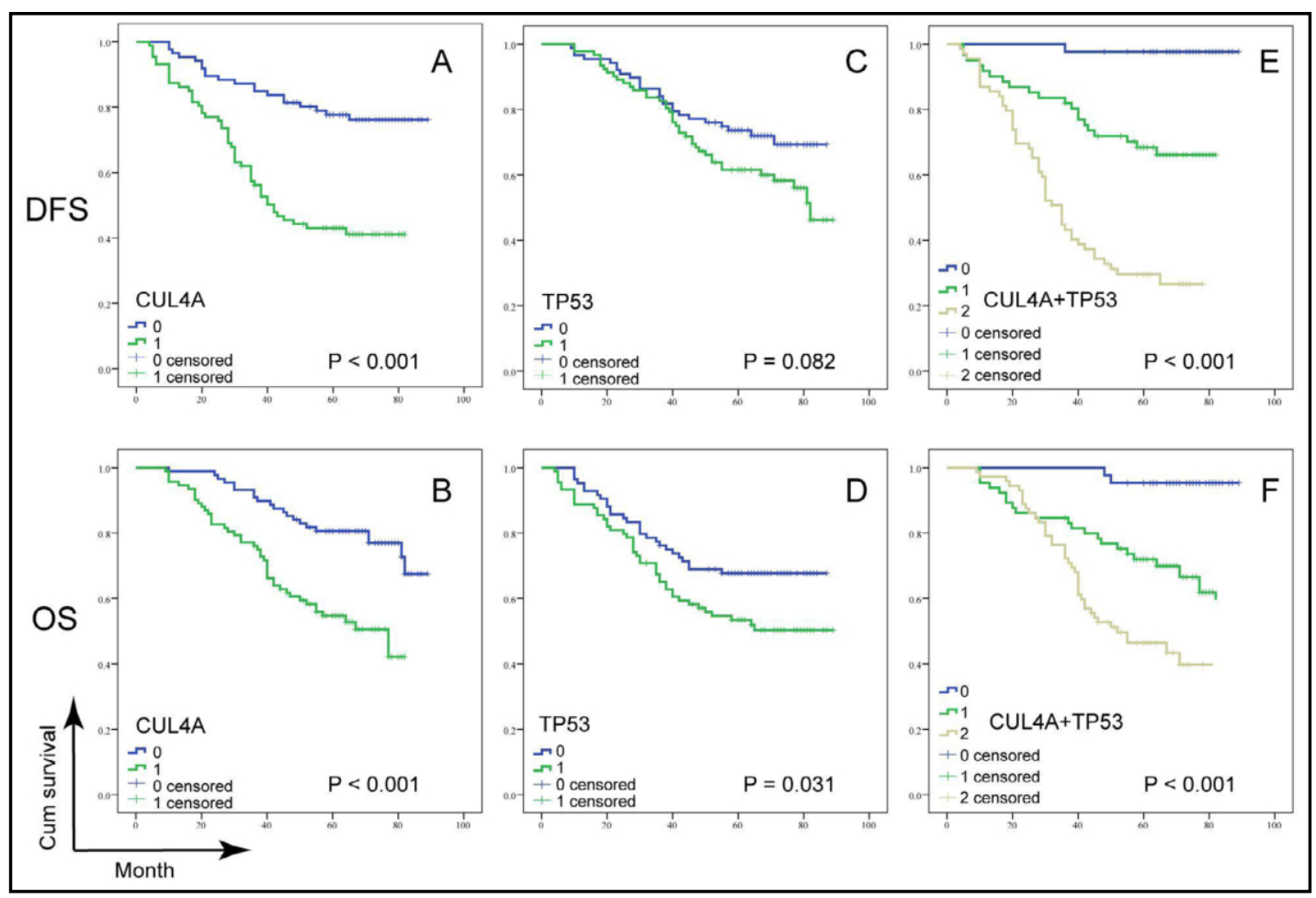

Fig. 2. Disease-free survival (DFS) and overall survival (OS) of colorectal cancer by the Kaplan-Meier method and the log-rank test. (A and B) DFS and OS curves of CUL4A+ (green line, 1) and CUL4A- (blue line, 0); (C and D) DFS and OS curves of TP53+ (green line, 1) and TP53- (blue line, 0); (E and F) DFS and OS curves of CUL4A+/TP53+ (brown line, 2), CUL4A+/TP53- or CUL4A-/TP53+ (green line, 1), CUL4A-/ TP53- (blue line, 0).

TP53 alone was not a prognostic indicator, there was a trend toward a better OS and DFS in the patient group with nonCUL4A+TP53+ tumors than that in the patient group with CUL4A+ or TP53+ tumors $(\mathrm{P}<0.001$, Fig. 2E and 2F). High CUL4A and high TP53 expression (CUL4A+/TP53+) was found to be a significant independent prognostic factor for DFS (HR 3.45; 95\% [CI], 2.08-5.73; P < 0.001) and OS (HR 2.29; 95\% CI, 1.29-4.17; $\mathrm{P}<0.001$ ) in multivariate analysis.

\section{CUL4A promotes colon cancer cell proliferation}

To examine the effect of CUL4A on colon cancer in vitro, colony formation assay related to cell proliferation was performed. The efficiency of SiRNA-mediated knockdown in HCT8 or HT29 cells was more than 70\%(Fig. 3A). As the immunohistochemical analysis showed (Fig. 3B), lentiviral vector transfer resulted in a decrease of CUL4A protein in HCT8 tumor xenografts. Colony formation assay showed that CUL4A knockdown inhibited cancer cell colonogenicity compared with control groups (Fig. 3C). Furthermore, we investigated the role of CUL4A in the growth of xenograft mouse model. Control cells and SiCUL4A cells were subcutaneously injected into nude mice ( $\mathrm{n}=5$ /group). The tumor growth curves and harvested tumor weights are shown in Fig. 3B. The tumors formed from SiCUL4A cells grew 


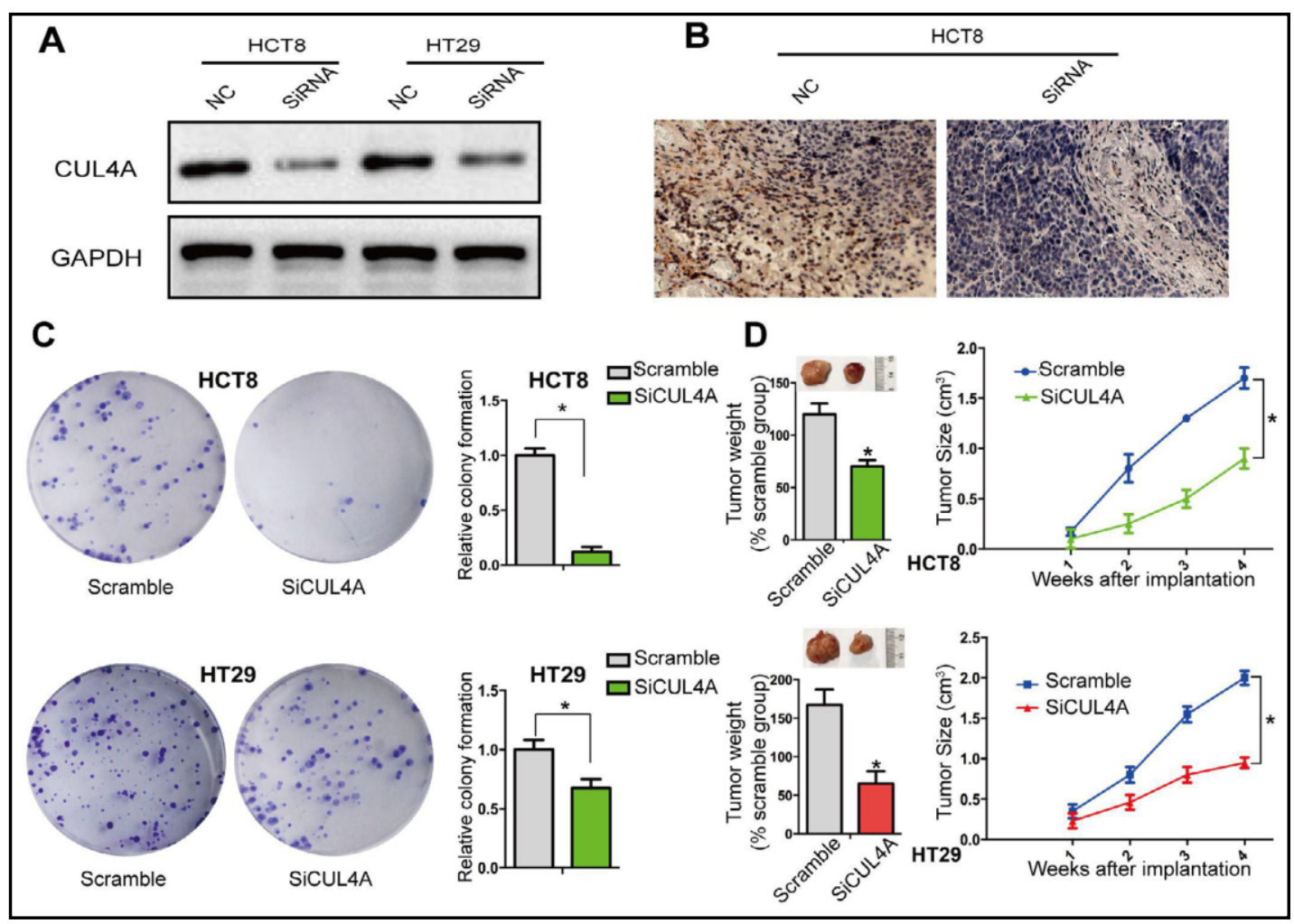

Fig. 3. CUL4A suppresses proliferation of colorectal cancer cells in vitro and in vivo. (A)The CUL4A knockdown effects were confirmed by WB in HCT8 and HT29 cell lines. GAPDH was used as an internal control. (B) Immunohistochemical staining for CUL4A expression in HCT8 tumor xenografts $(\times 200)$. (C)Effects of CUL4A knockdown on cell growth in vitro. (D) Representative Data of xenograft tumors in different treatment nude mice. Tumor volume and weight of xenograft tumors in nude mice were harvested in different treatment groups.

slower than those formed from the control cells (Fig. 3D). After 4 weeks, the volume of tumors induced by the CUL4A-suppressed cells was significantly reduced when compared to that induced by control cells (Fig. 3D).

CUL4A promotes migratory and invasive ability of colon cancer by reversing EMT tendency

In transwell cell invasion assays, representative data showed that SiCUL4A dramatically decreased the invasive ability of colorectal cells (Fig. 4A). These data suggest that CUL4A plays an important role in cell invasion. The SiCUL4A cells was wounded by scratching and maintained at $37^{\circ} \mathrm{C}$ for $24 \mathrm{~h}$. The knockdown of CUL4A attenuated the flattening and spreading of HCT8 and HT29 cells (Fig. 4B), indicating that CUL4A may have a significant effect on cell migration and invasion.

Since altered CUL4A expression was associated with the migratory and invasive ability of colon cancer, we subsequently investigated the capability of CUL4A to induce EMT in human colon cancer cells. We transfected CUL4A-SiRNA and the control vector into HCT8 and HT29 cells. We found that down-regulation of CUL4A in HCT8 and HT29 cells significantly decreased the levels of vimentin and $\mathrm{N}$-cadherin but increased E-cadherin expression in protein level (Fig. 4C). These results indicate that CUL4A promotes migratory and invasive ability of colon cancer by reversing EMT induction. 


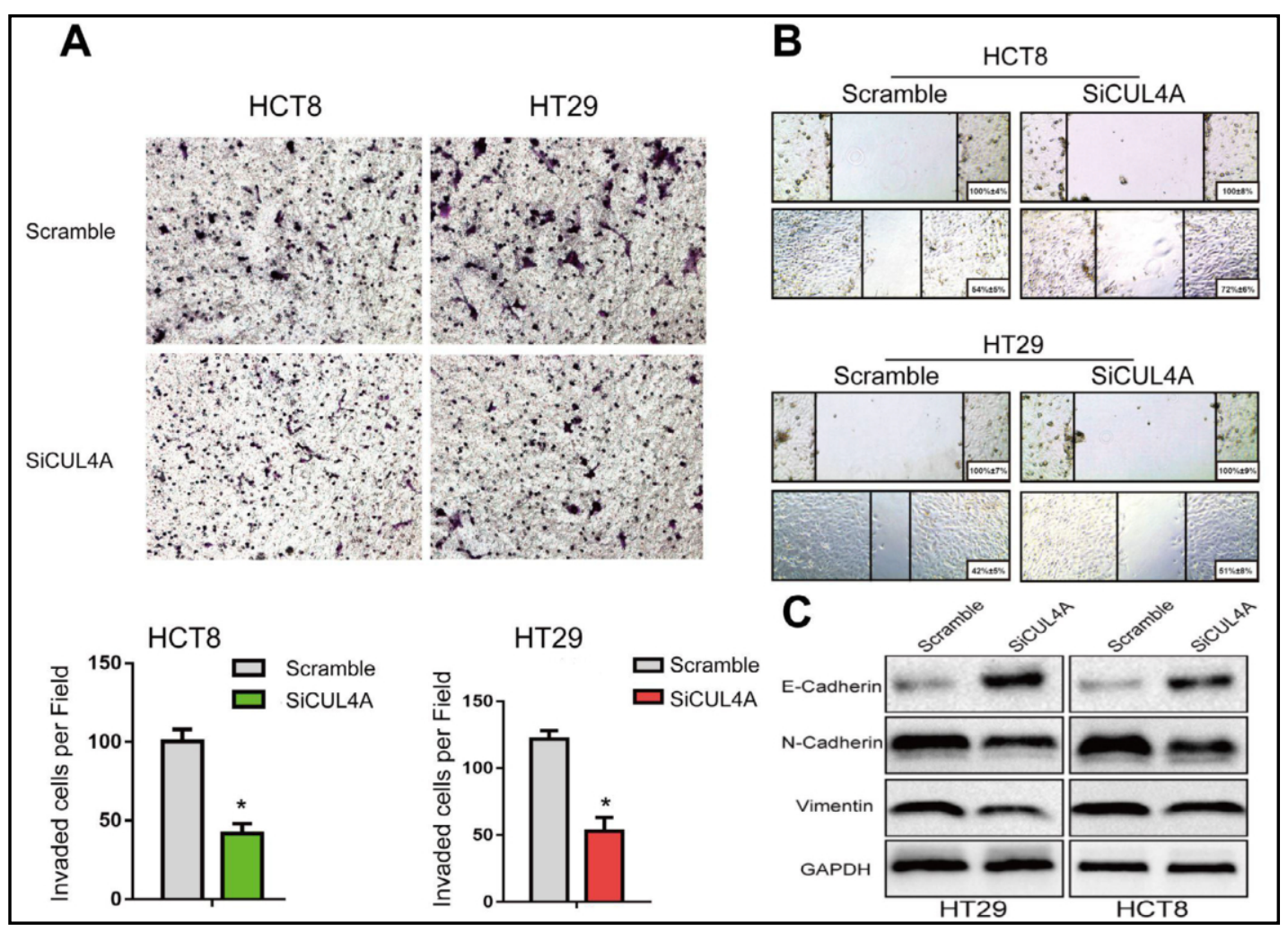

Fig. 4. Influence of CUL4A expression on colorectal cancer cell invasive ability and cell migration ability. (A) Transwell invasion assay with matrigel was performed in HCT8 and HT29 transfected with SiCUL4A. Magnification, 200×. (B) The wound healing migration assay was carried out in HCT8 and HT29 transfected with SiCUL4A. Magnification, 200×. (C) Western blot analysis of EMT markers. Down-regulated expression of CUL4A in HCT8 and HT29 cells significantly decreased the levels of vimentin and N-cadherin but increased E-cadherin expression in protein level.

\section{CUL4A activates the EMT via ZEB1}

Various transcriptional factors partake in regulating the EMT process. A prior study disclosed that ZEB1 is the transcriptional factor in the CUL4A-regulated EMT in breast cancer. In the interest of investigating the role of ZEB1 in the CUL4A-related EMT and metastasis, our study perfromed an analysis of the phenotypic changes in CUL4A overexpressing HCT8 cells with ZEB1 knockdown. To start with, ZEB1 expression is analysed through IHC assay, which showed that ZEB1 was overexpressed in primary tumor tissues in comparison with tumor adjacent tissues (Fig. 5A). On top of that, the active correlation between ZEB1 expression and CUL4A expression was affirmed (Fig. 5B). In addition, it was proved that ZEB1 expression was negatively correlated with E-cadherin expression (Fig. 5C). In vitro assays, ZEB1 expression was also negatively with E-cadherin expression and positively correlated with vimentin (Fig. $5 \mathrm{G})$. Moreover, the down-regulated expression of ZEB1 significantly inhibited the migratory and invasive abilities in HCT8 cell line (Fig. 5H and K). ZEB1 deficiency evidently reversed the decreased expression of E-cadherin and augmented the expression of vimentin triggered by CUL4A overexpression (Fig. 5D). Furthermore, the improved migratory and invasive abilities caused by CUL4A overexpression were also restrained by ZEB1 knockdown in the HCT8 cell line (Fig. 5E and F). Consequently, these discoveries indicated that ZEB1 regulates the CUL4A-induced EMT, migration and invasion in colorectal cancer. 
Fig. 5. ZEB1 mediates the metastasis regulated by CUL4A in colorectal cancer. (A) Representation of ZEB1 expression in colorectal normal and CRC tissues. (B) Linear regression analyses of IHC between CUL4A and ZEB1 expression in CRC. (C) Linear regression analyses of IHC between E-cadherin and ZEB1. (D) E-cadherin and vimentin expression after ZEB1 interference in CUL4A overexpres s in g HCT8 cells. (E and F) Migration and invasion ability in CUL4A overexpressing HCT8 cells with ZEB1 depletion. (G) CUL4A, E-CAD and Vimentin expression in SiZEB1 HCT8 cells. ( $\mathrm{H}$ and K) Migration and invasion ability in SiZEB1 HCT8 cells.

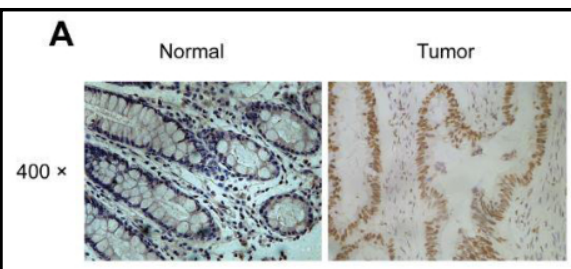

B

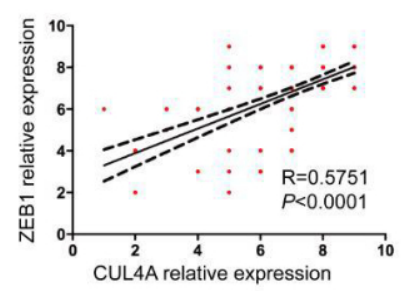

D

E

pLenti-CUL4A - + + +

$\begin{array}{lllll}\text { SiNC } & - & - & + & - \\ \text { SiZEB1 } & - & - & - & + \\ \text { CUL4A } & & & & \end{array}$

ZEB1

E-CAD

Vimentin

$\beta$-actin

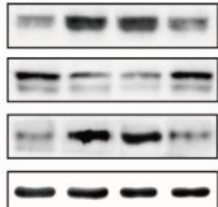

G
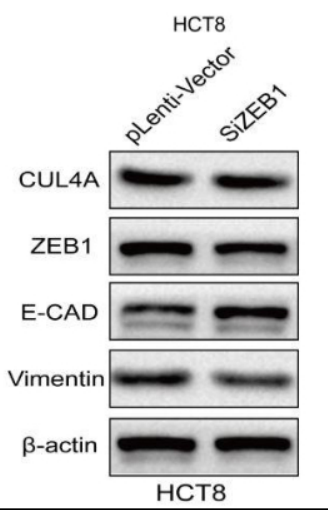

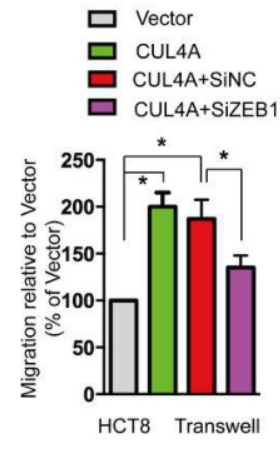

H

C
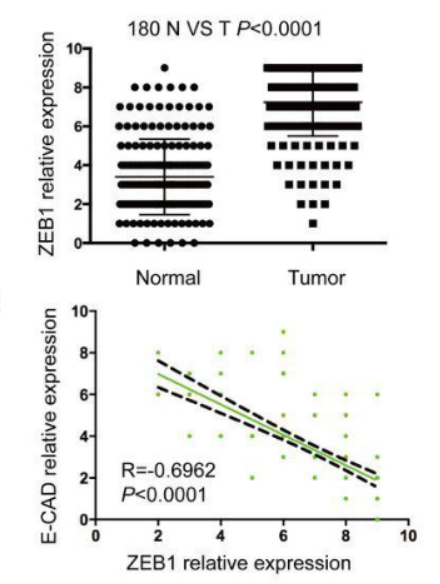

F
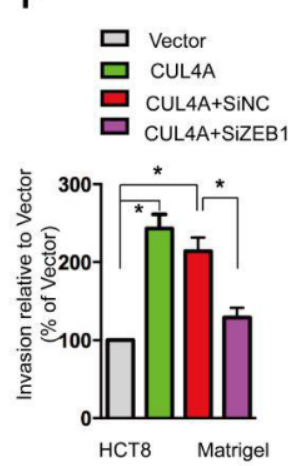

$\mathbf{K}$ $\square$ vector

口 SiZEB1

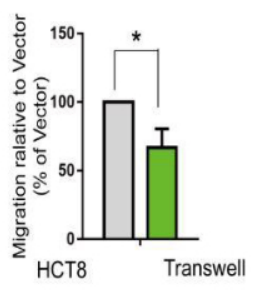

$\square$ Vector

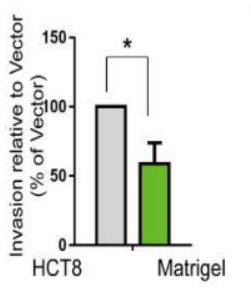

\section{Discussion}

CUL4A, a member of the cullin family of proteins, composes the multifunctional ubiquitin ligase E3 complex, is essential for the ubiquitination of several well-defined tumor suppressor genes such as p21 [33], p27 [34], and p53 [35]. Thus, CUL4A may act as an oncogene, but whether CUL4A plays a role in colorectal cancer remains unknown. Our study shows that (a) the expression of CUL4A is upregulated in colorectal cancer. (b) aberrant expression of CUL4A is significantly associated with tumor size, differentiation, $\mathrm{N}$ stage and AJCC stage. (c) high CUL4A

and high TP53 expression is a significant independent prognostic factor. (d) the overexpression of CUL4A promotes proliferation, migration and invasion of colon cancer. (e) CUL4A promotes the migration and invasion of colon cancer by affecting EMT induction.

In this study, we have determined CUL4A and TP53 protein expression in colorectal tissues by immunohistochemistry analysis on tissue microarray (TMA). We found that both CUL4A and TP53 protein expression were significantly higher in cancerous tissues 
than in normal tissues. In colorectal cancers, we found high CUL4A protein expression was associated with tumor size, differentiation, N stage and AJCC stage. We also detected significant correlation between CUL4A and TP53 expression. In multivariate analysis, we found high CUL4A expression was significantly associated with patients' poor DFS and OS. Our data indicated that high TP53 expression was associated with poor overall survival but not disease free survival in colorectal cancer.

Multivariate analysis showed that CUL4A expression alone or combined with mutant TP53 expression appeared to be an independent prognostic factor for OS and DFS in colon cancer. We proposed that data on CUL4A and mutant TP53 expression in tumors could be used to design optimal, individualized treatment and to help identify patients who may or may not benefit from close monitoring after surgery. Most of all, the mutant TP53, a gene significantly correlated with colon cancer, although not predictive of cancer prognosis by itself, could be of strong predictive value when evaluated with CUL4A expression. In summary, this study provided critical insight into the role of the CUL4A gene in the progression of colon cancer. The frequent up-regulation of CUL4A expression in human colon cancer highlights its potential as a novel therapeutic target for this cancer. These findings might be helpful in designing future studies to understand the molecular development of colon cancer.

Researchers have found that CUL4A plays a critical role in cellular proliferation and tumor invasion [36-38]. At the same time, our experiments in vivo and in vitro support those clinical assays. Ectopic CUL4A expression affected proliferation, migratory and invasion of colorectal cancer. CUL4A, which is the core structure in the main skeleton of ubiquitin ligase E3, can degrade various proteins by ubiquitin-proteasome system (UPS) to regulate the level and function of proteins. Thus, CUL4A-DDB1-ROC1 complex plays an important role in the occurrence and development of malignant tumors. CUL4A-DDB1 can regulate the methylation of H3K4 by the ubiquitin hydrolysis of WDR5 and RBBP5, which leads to the activation and silencing of some genes. Study have shown that CUL4A can induce the activation of ZEB1 through regulating H3K4 trimethylation, which is a mediator for CUL4Ainduced EMT, migration, invasion, and tumor metastasis in breast cancer [36]. It was reported that the level of P53 protein was regulated by both MDM2 and CUL4A-DDB1-ROC1 complex. The deletion of DDB1 leads to the accumulation of P53, while ectopic expression of CUL4A decreased the level of P53 [39]. In our study, knockdown of CUL4A attenuated proliferation, migratory and invasive abilities of colorectal cancer cells in vitro and tumorigenicity in vivo.

Although the understanding of cell migration and invasion has evolved greatly, mechanisms involved in the progression of colorectal cancer are not fully understood. Convincing evidence suggests that the EMT has a close relationship with metastasis and invasion of malignant tumor [40-42]. In the current study, we found that expression of CUL4A is significantly associated with the EMT. Deregulated CUL4A expression increased levels of epithelial markers (E-cadherin), and decreased levels of mesenchymal markers (N-cadherin and vimention). ZEB1 plays a vital role as regulatory molecule in the process of carcinoma cells epithelial-mesenchymal transition (EMT), which brings about tumor invasion and metastasis. Besides, this report showed that CUL4A could regulate EMT via ZEB1 in colorectal cancer cell lines. Moreover, ZEB1 interference noticeably cut down the metastasis and EMT result from CUL4A overexpression in HCT8 cells.

\section{Conclusion}

To our best knowledge, this is the first study investigating epithelial CUL4A protein expression as well as potential CUL4A and TP53 protein interaction in colorectal cancers. Our study has several limitations: first, it is a retrospective observational study, the conclusions might not be applicable to the general population. Larger prospective studies are needed to confirm our findings. Secondly, the roles of several transcription factors and non-coding RNA as EMT regulators (miR-137 and miR-9) have not been explored [37, 43, 44]. Thirdly, the relationship between CUL4A and TP53 has not been further explored. Further studies 
are needed to explore the special molecular mechanisms that influence the expression and metastasis function of CUL4A. In conclusion, we have shown that the critical role of CUL4A gene in the progression of colorectal cancer, and is the first to discover its relationship with EMT in colorectal cancer via, at least partially, up-regulating transcriptional regulation factor ZEB1. High CUL4A protein expression is an independent prognostic marker in colorectal cancers. The findings reported here also indicate that CUL4A overexpression in combination with mutant TP53 protein accumulation was associated with recurrent cancer and a poor survival rate. CUL4A may be a therapeutic target for colorectal cancer.

\section{Acknowledgements}

This work was supported in part by National Natural Science Foundation of China (No. 81472284 and 81672699), Shanghai Program for Young Top Talents and Shanghai Pujiang Program (No. 16PJD004) and Shanghai Young Talent Program.

\section{Disclosure Statement}

The authors declare no competing financial interests.

\section{References}

1 Siegel R, Desantis C, Jemal A: Colorectal cancer statistics, 2014 CA Cancer. J Clin 2014;64:104-117.

-2 Venook AP, Niedzwiecki D, Lopatin M, Ye X, Lee M, Friedman PN, Frankel W, Clark-Langone K, Millward C, Shak S, Goldberg RM, Mahmoud NN, Warren RS, Schilsky RL, Bertagnolli MM: Biologic determinants of tumor recurrence in stage II colon cancer: validation study of the 12-gene recurrence score in cancer and leukemia group B (CALGB) 9581.J Clin Oncol 2013;31:1775-1781.

-3 Kucukzeybek Y, Dirican A, Demir L, Yildirim S, Akyol M, Yildiz Y, Bayoglu IV, Alacacioglu A, Varol U, Salman T, Yildiz I, Can H, Tarhan MO: Adjuvant chemotherapy and prognostic factors in stage II colon cancer--Izmir Oncology Group Study. Asian Pac J Cancer Prev 2015;16:2413-2418.

4 Yothers G, O'Connell MJ, Lee M, Lopatin M, Clark-Langone KM, Millward C, Paik S, Sharif S, Shak S, Wolmark N: Validation of the 12-gene colon cancer recurrence score in NSABP C-07 as a predictor of recurrence in patients with stage II and III colon cancer treated with fluorouracil and leucovorin (FU/LV) and FU/LV plus oxaliplatin. J Clin Oncol 2013;31:4512-4519.

5 Schepeler T, Reinert JT, Ostenfeld MS, Christensen LL, Silahtaroglu AN, Dyrskjot L, Wiuf C, Sorensen FJ, Kruhoffer M, Laurberg S, Kauppinen S, Orntoft TF and Andersen CL: Diagnostic, prognostic microRNAs in stage II colon cancer. Cancer Res 2008;68:6416-6424.

6 Tian S, Simon I, Moreno V, Roepman P, Tabernero J, Snel M, van't Veer L, Salazar R, Bernards R, Capella G: A combined oncogenic pathway signature of BRAF, KRAS and PI3KCA mutation improves colorectal cancer classification and cetuximab treatment prediction. Gut 2013;62:540-549.

7 Rustgi AK: BRAF: a driver of the serrated pathway in colon cancer. Cancer cell 2013;24:1-2.

-8 Gill S, Loprinzi CL, Sargent DJ, Thome SD, Alberts SR, Haller DG, Benedetti J, Francini G, Shepherd LE, Francois Seitz J, Labianca R, Chen W, Cha SS, Heldebrant MP, Goldberg RM: Pooled analysis of fluorouracil-based adjuvant therapy for stage II and III colon cancer: who benefits and by how much? J Clin Oncol 2004;22:1797-1806.

-9 Zhong W, Feng H, Santiago FE, Kipreos ET: CUL-4 ubiquitin ligase maintains genome stability by restraining DNA-replication licensing. Nature 2003;423:885-889.

10 Higa LA, Wu M, Ye T, Kobayashi R, Sun H, Zhang H: CUL4-DDB1 ubiquitin ligase interacts with multiple WD40-repeat proteins and regulates histone methylation. Nat Cell Biol 2006;8:1277-1283.

11 Katiyar S, Liu E, Knutzen CA, Lang ES, Lombardo CR, Sankar S, Toth JI, Petroski MD, Ronai Z, Chiang GG: REDD1, an inhibitor of mTOR signalling, is regulated by the CUL4A-DDB1 ubiquitin ligase. EMBO Rep 2009;10:866-872. 


\section{Cellular Physiology Cell Physiol Biochem 2018;51:2829-2842 and Biochemistry \begin{tabular}{l|l}
$\overline{\mathrm{DOI}}$ : 10.1159/000496013 & $\begin{array}{l}\text { (c) } 2018 \text { The Author(s). Published by S. Karger AG, Basel } \\
\text { www.karger.com/cpb }\end{array}$
\end{tabular}

12 Matsuda N, Azuma K, Saijo M, Iemura S, Hioki Y, Natsume T, Chiba T, Tanaka K, Tanaka K: DDB2, the xeroderma pigmentosum group E gene product, is directly ubiquitylated by Cullin 4A-based ubiquitin ligase complex. DNA Repair (Amst) 2005;4:537-545.

13 Higa LA, Yang X, Zheng J, Banks D, Wu M, Ghosh P, Sun H, Zhang H: Involvement of CUL4 ubiquitin E3 ligases in regulating CDK inhibitors Dacapo/p27Kip1 and cyclin E degradation. Cell Cycle 2006;5:71-77.

14 Gupta A, Yang LX, Chen L. Study of the G2/M cell cycle checkpoint in irradiated mammary epithelial cells overexpressing Cul-4A gene: Int J Radiat Oncol Biol Phys 2002;52:822-830.

15 Waning DL, Li B, Jia N, Naaldijk Y, Goebel WS, HogenEsch H, Chun KT: Cul4A is required for hematopoietic cell viability and its deficiency leads to apoptosis. Blood 2008;112:320-329.

-16 Melchor L, Saucedo-Cuevas LP, Munoz-Repeto I, Rodriguez-Pinilla SM, Honrado E, Campoverde A, Palacios J, Nathanson KL, Garcia MJ, Benitez J: Comprehensive characterization of the DNA amplification at 13q34 in human breast cancer reveals TFDP1 and CUL4A as likely candidate target genes. Breast Cancer Res 2009;11:R86.

17 Yasui K, Arii S, Zhao C, Imoto I, Ueda M, Nagai H, Emi M, Inazawa J: TFDP1, CUL4A, and CDC16 identified as targets for amplification at 13q34 in hepatocellular carcinomas. Hepatology. 2002;35:1476-1484.

18 Wang Y, Zhang P, Liu Z, Wang Q, Wen M, Wang Y, Yuan H, Mao JH, Wei G: CUL4A overexpression enhances lung tumor growth and sensitizes lung cancer cells to erlotinib via transcriptional regulation of EGFR. Mol Cancer 2014;13:252.

19 Ren S, Xu C, Cui Z, Yu Y, Xu W, Wang F, Lu J, Wei M, Lu X, Gao X, Liang Y, Mao JH, Sun Y: Oncogenic CUL4A determines the response to thalidomide treatment in prostate cancer. J Mol Med (Berl) 2012;90:11211132.

20 Birner P, Schoppmann A, Schindl M, Dinhof C, Jesch B, Berghoff AS, Schoppmann SF: Human homologue for Caenorhabditis elegans CUL-4 protein overexpression is associated with malignant potential of epithelial ovarian tumours and poor outcome in carcinoma. J Clin Pathol 2012;65:507-511.

21 Moriya M, Slade N, Brdar B, Medverec Z, Tomic K, Jelakovic B, Wu L, Truong S, Fernandes A, Grollman AP: TP53 Mutational signature for aristolochic acid: an environmental carcinogen. Int J Cancer 2011;129:1532-1536.

22 Gluck S, Ross JS, Royce M, McKenna EF, Jr., Perou CM, Avisar E, Wu L: TP53 genomics predict higher clinical and pathologic tumor response in operable early-stage breast cancer treated with docetaxelcapecitabine +/- trastuzumab. Breast Cancer Res Treat. 2012;132:781-791.

23 Cooks T, Pateras IS, Tarcic O, Solomon H, Schetter AJ, Wilder S, Lozano G, Pikarsky E, Forshew T, Rosenfeld N, Harpaz N, Itzkowitz S, Harris CC, Rotter V, Gorgoulis VG, Oren M: Mutant p53 prolongs NFkappaB activation and promotes chronic inflammation and inflammation-associated colorectal cancer. Cancer cell 2013;23:634-646.

24 Levy A, Hall L, Yeudall WA, Lightman SL: p53 gene mutations in pituitary adenomas: rare events. Clin Endocrinol (Oxf) 1994;41:809-814.

-25 Brosh R, Rotter V: When mutants gain new powers: news from the mutant p53 field. Nat Rev Cancer 2009;9:701-713.

26 Brooks CL, Gu W: p53 ubiquitination: Mdm2 and beyond. Mol Cell. 2006;21:307-315.

-27 Goldstein I, Marcel V, Olivier M, Oren M, Rotter V, Hainaut P: Understanding wild-type and mutant p53 activities in human cancer: new landmarks on the way to targeted therapies. Cancer Gene Ther 2011;18:2-11.

-28 Frum RA, Grossman SR: Mechanisms of mutant p53 stabilization in cancer. Subcell Biochem 2014;85:187-197.

29 Santoro R, Strano S, Blandino G: Transcriptional regulation by mutant p53 and oncogenesis. Subcell Biochem 2014;85:91-103.

-30 Weissmueller S, Manchado E, Saborowski M, Morris JPt, Wagenblast E, Davis CA, Moon SH, Pfister NT, Tschaharganeh DF, Kitzing T, Aust D, Markert EK, Wu J, Grimmond SM, Pilarsky C, Prives C: Mutant p53 drives pancreatic cancer metastasis through cell-autonomous PDGF receptor beta signaling. Cell 2014;157:382-394.

31 Levine AJ, Oren M: The first 30 years of p53: growing ever more complex. Nat Rev Cancer 2009;9:749758.

-32 Miyoshi A, Kitajima Y, Sumi K, Sato K, Hagiwara A, Koga Y, Miyazaki K: Snail and SIP1 increase cancer invasion by upregulating MMP family in hepatocellular carcinoma cells. Br J Cancer 2004;90:1265-1273. 


\section{Cellular Physiology Cell Physiol Biochem 2018;51:2829-2842

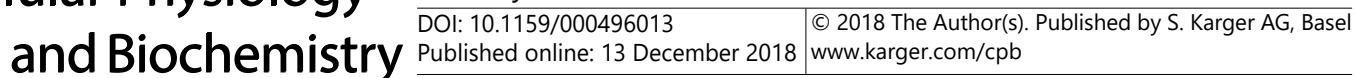 \\ Li et al.: CUL4A and TP53 in Colorectal Cancer}

33 Nishitani H, Shiomi Y, Iida H, Michishita M, Takami T, Tsurimoto T: CDK inhibitor p21 is degraded by a proliferating cell nuclear antigen-coupled Cul4-DDB1Cdt2 pathway during S phase and after UV irradiation. J Biol Chem 2008;283:29045-29052.

34 Li B, Jia N, Kapur R, Chun KT: Cul4A targets p27 for degradation and regulates proliferation, cell cycle exit, and differentiation during erythropoiesis. Blood 2006;107:4291-4299.

-35 Nag A, Bagchi S, Raychaudhuri P: Cul4A physically associates with MDM2 and participates in the proteolysis of p53. Cancer Res 2004;64:8152-8155.

-36 Wang Y, Wen M, Kwon Y, Xu Y, Liu Y, Zhang P, He X, Wang Q, Huang Y, Jen KY, LaBarge MA, You L, Kogan SC, Gray JW, Mao JH, Wei G: CUL4A induces epithelial-mesenchymal transition and promotes cancer metastasis by regulating ZEB1 expression. Cancer Res 2014;74:520-531.

-37 Deng J, Lei W, Xiang X, Zhang L, Lei J, Gong Y, Song M, Wang Y, Fang Z, Yu F, Feng M, Sun Z, Chen J, Zhan Z, Xiong J: Cullin 4A (CUL4A), a direct target of miR-9 and miR-137, promotes gastric cancer proliferation and invasion by regulating the Hippo signaling pathway. Oncotarget 2016;7:10037-10050.

-38 Pan Y, Wang B, Yang X, Bai F, Xu Q, Li X, Gao L, Ma C, Liang X: CUL4A facilitates hepatocarcinogenesis by promoting cell cycle progression and epithelial-mesenchymal transition. Sci Rep 2015;5:17006.

39 Banks D, Wu M, Higa LA, Gavrilova N, Quan J, Ye T, Kobayashi R, Sun H, Zhang H: L2DTL/CDT2 and PCNA interact with p53 and regulate p53 polyubiquitination and protein stability through MDM2 and CUL4A/ DDB1 complexes. Cell Cycle 2006;5:1719-1729.

40 Zhang M, Song S, Yi Z, Zhao X, Fu L, Wang L, Ma C, Mao M, Xing Y, Zhu D: Human biliverdin reductase promotes EMT through the ERK1/2 signal pathway in breast cancer. Eur J Pharmacol 2016;788:45-53.

-41 Grassi ML, Palma CS, Thome CH, Lanfredi GP, Poersch A, Faca VM: Proteomic analysis of ovarian cancer cells during epithelial-mesenchymal transition (EMT) induced by epidermal growth factor (EGF) reveals mechanisms of cell cycle control. J Proteomics 2017;16:2-11.

42 Meng J, Zhang XT, Liu XL, Fan L, Li C, Sun Y, Liang XH, Wang JB, Mei QB, Zhang F, Zhang T: WSTF promotes proliferation and invasion of lung cancer cells by inducing EMT via PI3K/Akt and IL-6/STAT3 signaling pathways. Cell Signal 2016;28:1673-1682.

43 Han Y, Bi Y, Bi H, Diao C, Zhang G, Cheng K, Yang Z: miR-137 suppresses the invasion and procedure of EMT of human breast cancer cell line MCF-7 through targeting CtBP1. Hum Cell 2016;29:30-36.

44 Yu JM, Sun W, Hua F, Xie J, Lin H, Zhou DD, Hu ZW: BCL6 induces EMT by promoting the ZEB1-mediated transcription repression of E-cadherin in breast cancer cells. Cancer Lett 2015;365:190-20. 